\title{
Comparison of Two Restoration Techniques in the Context of 3D Medical Imaging*
}

\author{
Miguel A. Rodriguez-Florido ${ }^{1}$, Karl Krissian ${ }^{2}$, Juan Ruiz-Alzola ${ }^{1}$, and \\ Carl-Fredrik Westin ${ }^{3}$ \\ 1 Departamento de Señales y Comunicaciones \\ 2 Departamento de Informática y Sistemas \\ Universidad de Las Palmas de Gran Canaria - Las Palmas, Spain \\ \{marf, jruiz\}@dsc.ulpgc.es ; krissian@dis.ulpgc.es \\ 3 Surgical Planning Lab. - BWH - Harvard Medical School - Boston(MA) - USA \\ westin@bwh.harvard.edu
}

\begin{abstract}
In this paper, we compare two restoration techniques applied to 3D angiographies and to femoral CT scans. The first technique uses a Partial Derivative Equation and the second one is based on an extension of adaptive Wiener filters. We first present each method. Then, we discuss and compare the estimation of the local orientations in 3D images obtained either by the smoothed gradient and the principal curvature directions or by the eigenvectors of the structure tensor. A good estimation of the orientations is essential because it directs the restoration process. Finally, we compare the restored images on both synthetic and real images for the two studied applications.
\end{abstract}

\section{Introduction}

Image enhancement is especially important in medical imaging because it allows physicians to obtain a better visual interpretation, especially when viewing weak structures (i.e. thin vessels), differentianting false joint regions (i.e. space between femoral head and hip) and in other numerous clinical applications. Moreover, enhancement is a preprocessing step for subsequent automated medical analysis, such as segmentation of different tissues or registration of images from different modalities. In this sense, this paper presents a qualitative comparison between anisotropic diffusion [5, 9, 11] and anisotropic adaptive frequency filtering [4, 13], that will permit future algorithmic improvements or a feedback process between both. We also compare the local structure estimation that each technique uses, analyzing which one is the best in different cases. The paper is structured as follows: first, we present a brief description of both restoration methods, second we compare two orientation extraction techniques, and finally we present our results.

\footnotetext{
* This work was partially funded by the European project ERB-4061-PL-97-0777, by European Comission and Spanish Gov. joint research grant 1FD97-0881-C02-01, and by CIMIT and NIH P41-RR13218. The first author is funded by a FPU grant at the University of Las Palmas de Gran Canaria.
} 


\section{Presentation of the Two Methods}

In this section, we present two 3D anisotropic filtering techniques. The first is based on anisotropic diffusion and uses the gradient and the principal curvature directions. The second technique is designed in the Fourier domain and uses the eigenvectors of the structure tensor to drive the filtering.

\subsection{Flux-Based Anisotropic Diffusion}

In [5, 6], a multi-directional flux-based diffusion scheme is proposed. The general expression of the diffusion equation is:

$$
\left\{\begin{array}{l}
u(x, 0)=u_{0} \\
\frac{\partial u}{\partial t}=\operatorname{div}(\mathbf{F})+\beta\left(u_{0}-u\right) .
\end{array}\right.
$$

where $\mathbf{F}$ is the diffusion flux that drives the diffusion and $\beta$ is a data attachment term which allows a convergence of the diffusion scheme to an image $u$ that remains close to the initial data $u_{0}$. The expression of the differential equation as the divergence of a vector field ensures conservation of image intensity. Particular cases of this equation with $\beta=0$ are:

- the heat diffusion equation $\mathbf{F}=\nabla u$ which is equivalent to a Gaussian convolution that reduces noise, but does not preserve the contours of the image, smoothing the information in an isotropic way.

- the Perona and Malik equation [9] with $\mathbf{F}=g(\|\nabla u\|) \nabla u$ where $g$ is a diffusion function that reduces the diffusion for "high" gradients. To achieve this goal, a threshold $\delta$ on the norm of the gradient is introduced. $g$ is chosen in such a way that it diffuses a little when $\|\nabla u\|$ is higher than $\delta$; it tends to a positive constant when $\|\nabla u\|$ is close to zero, acting like heat diffusion. However, the flux is always oriented in the gradient direction.

- the matrix diffusion, developed by Weickert [11, uses a matrix diffusion $D$ with a flux $\mathbf{F}=D \nabla u$. Then the flux can be expressed as $\mathbf{F}=D \nabla u=\sum_{i=0}^{2} \lambda_{i} u_{\mathbf{v}_{i}} \mathbf{v}_{i}$ with $\lambda_{i}$ and $\mathbf{v}_{i}$ the eigenvalues and eigenvectors of $D$ and $u_{\mathbf{v}_{i}}=\nabla u \cdot \mathbf{v}_{i}$ is the first order derivative of the intensity in the direction of $\mathbf{v}_{i}$.

Let $\left(\mathbf{e}_{0}, \mathbf{e}_{1}, \mathbf{e}_{2}\right)$ denote any orthogonal unit basis of $\mathbb{R}^{3}$ that generally depends on the local structures of the image. Then, the diffusion flux proposed in [5, 6] for this basis is written as:

$$
\mathbf{F}=\sum_{i=0}^{2} \phi_{i}\left(u_{\mathbf{e}_{i}}\right) \mathbf{e}_{i} .
$$

It is equivalent to tensor diffusion where the eigenvectors of the diffusion matrix are $\left(\mathbf{e}_{i}\right)_{i \in\{0,1,2\}}$ and the eigenvalues are functions of the first order derivative of the intensity in the direction of the associated eigenvector: $\lambda_{i}=\lambda_{i}\left(u_{\mathbf{e}_{i}}\right)$ leading to diffusion functions $\phi_{i}\left(u_{\mathbf{e}_{i}}\right)=\lambda_{i}\left(u_{\mathbf{e}_{i}}\right) u_{\mathbf{e}_{i}}$. This choice allows the separation of diffusion in different directions so that the diffusion in a given direction does not depend on the intensity variations in the other directions. Some interpretation of this diffusion scheme is given in [5, 6, 7]. 
For the local orientations, we use the gradient and principal curvature directions computed on the smoothed image $u^{*}$, where the smoothing is obtained by convolution with a Gaussian of standard deviation $\sigma$. This basis corresponds respectively to unit vectors in the directions of the gradient $\left(\mathbf{e}_{0}=\frac{\nabla u^{*}}{\left\|\nabla u^{*}\right\|}\right)$, and of the maximal and minimal curvature of the smoothed image. The principal curvature directions can be computed as two of the eigenvalues (eigenvectors respectively) of the matrix $P H P$, where $H$ is the Hessian matrix of the image and $P$ is the projection matrix orthogonal to the gradient direction, that is $H^{\prime}=P H_{\sigma} P$ with $P=I-\mathbf{e}_{0} \mathbf{e}_{0}{ }^{t}$, where $H_{\sigma}$ is the Hessian matrix of the smoothed image previously computed and $I$ is the identity matrix. One of the eigenvectors of this matrix is the gradient of the smoothed image with a zero associated eigenvalue, and the two others are the directions of principal curvature.

The diffusion functions $\Phi_{i}$ of eq. (2) are chosen as $\Phi_{0}(x)=x e^{-\left(\frac{x}{\delta}\right)^{2}}$ with a threshold $\delta$ on the intensity derivative in the gradient direction, $\Phi_{1}(x)=0$ and $\Phi_{2}(x)=x \alpha$, where $\alpha$ is a positive constant allowing a diffusion only in the minimal curvature direction.

\subsection{Anisotropic Adaptive Filtering}

Here we describe a method based on an adaptive extension of the well-known Wiener filter. The adaptive filter is steered by means of a local estimation of structure as provided by an auxiliary bank of filters which enhances the local orientation and the degree of anisotropy [3, 4].

Consider the Wiener filter $H=\frac{S_{f f}}{S_{f f}+\sigma_{n}^{2}}$, where $\sigma_{n}$ is the noise standard deviation and $S_{f f}$ is the power density spectrum. Based on the conventional unsharp masking technique used by photographers 10, Abramatic and Silvermann [1] proposed to use the filter $H_{\alpha}=H+(1-\alpha)(1-H)$ in order to isotropically trade-off a low-pass and a high-pass component weighting them with a visibility function $0 \leq \alpha \leq 1$. Knutsson et al. [4] introduced an anisotropy component to this filter:

$$
H_{\alpha, \gamma}=H+(1-\alpha)\left(\gamma+(1-\gamma) \cos ^{2}(\phi-\theta)\right)(1-H)
$$

where $\gamma$ weights the degree of anisotropy of the filter and $\theta$ is the main orientation of the image local structure. The $\cos ^{2}(\phi-\theta)$ term is a polar function which shapes the high pass component of the filter preventing any smoothing along $\theta$. In order to generalize this idea to $\mathrm{N}$-dimensions, it is convenient to describe the local structure by means of a second order symmetric positive semidefinite tensor $\mathbf{T}$, which principal eigenvector is aligned along the main direction of the local structure. This can be visualized as a local ellipsoid elongated along such direction. Different approaches can be used to estimate the local structure tensor [2, 4, 12]. Here we combine the output from a bank of spherically separable quadrature filters; details can be found in [3, 12.

An extension to the 2-D filter for the $\mathrm{N}$-dimensional case is [13]:

$$
H_{\gamma}=H+(1-H)<\mathbf{C}, \mathbf{U}>
$$


where $\mathbf{C}=\sum_{k=1}^{N} \gamma_{k} \hat{\mathbf{e}}_{k} \hat{\mathbf{e}}_{k}^{T}$ is a control tensor, $\mathbf{u}$ is a vector of spatial frequencies, $\mathbf{U}=\hat{\mathbf{u}} \hat{\mathbf{u}}^{T}$ is the outer product of the unit frequencies and $\langle\mathbf{C}, \mathbf{U}\rangle$ is an inner product. The eigenvalues of the control tensor indicate the degree of smoothing along the associated directions and hence the control tensor must be related to the tensor structure.

In our implementation, the control tensor is obtained from $\mathbf{C}=m\left(\lambda_{1}\right) \mathbf{T}^{\prime}$, where $m$ is the mapping function

$$
m\left(\lambda_{1}\right)=\gamma\left(1-\lambda_{1}\right)+\frac{\lambda_{1}^{\beta}}{\lambda_{1}^{(\alpha+\beta)}+\sigma^{\beta}},
$$

$\mathbf{T}^{\prime}$ is the local structure tensor normalized by the globally largest eigenvalue over the whole dataset, and $\lambda_{1}$ is the maximum eigenvalue of $\mathbf{T}^{\prime}$ for every voxel. Details can be found in 13 .

\section{Orientation Extraction}

In this section we compare on $3 \mathrm{D}$ synthetic images the orientations obtained by the gradient of the smoothed image $\mathbf{e}_{g}$ and the maximal and minimal curvature directions $\mathbf{e}_{M}$ and $\mathbf{e}_{m}$, with the orientations obtained by the eigenvectors of the structure tensor, denoted by $\mathbf{e}_{\lambda_{1}}, \mathbf{e}_{\lambda_{2}}, \mathbf{e}_{\lambda_{3}}$, and ordered decreasingly according to associated eigenvalues.
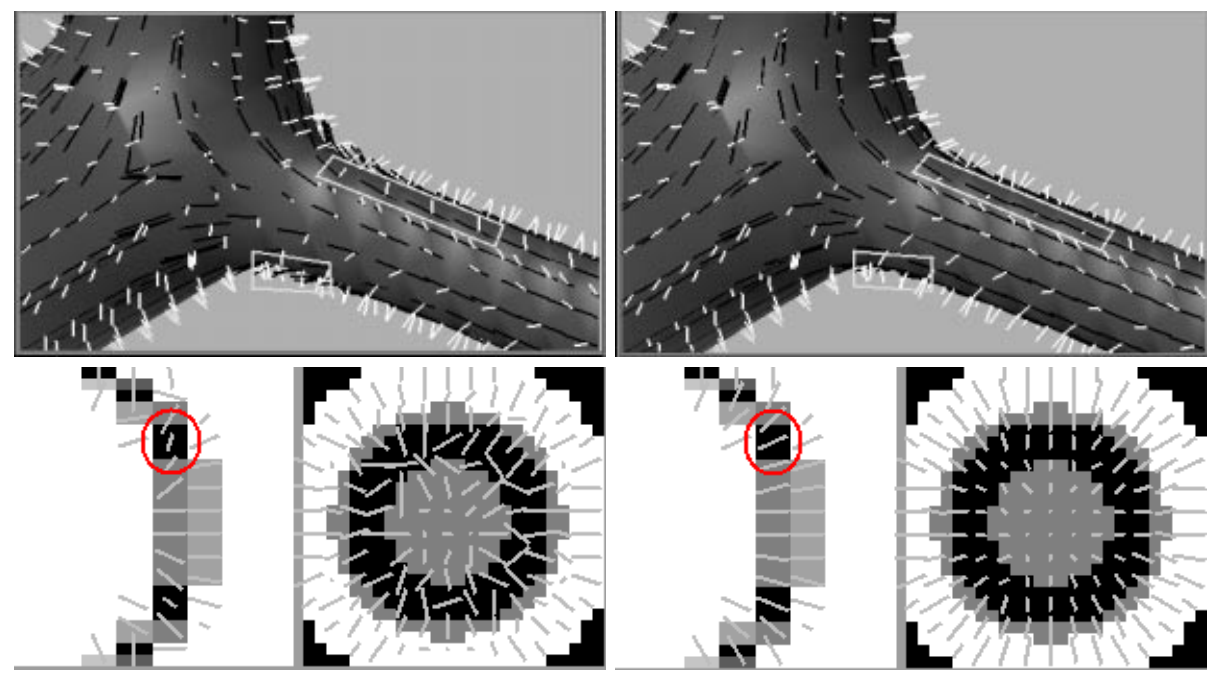

Fig. 1. Top row, comparison of the estimated orientations on a synthetic 3D junction. Bottom row, comparison of the estimated orientations on a synthetic 3D joint, where each image on the left represents a YZ slice, and on the right, an XY slice. The left column shows orientation obtained by the gradient and the minimal curvature, and the right column shows the first and the last eigenvectors of the structure tensor. 
We use two synthetic 3D images to test these orientations. The first one is a synthetic 3D junction of vessels and the second one is a synthetic $3 \mathrm{D}$ hip joint (fig. 3). The intensity of the synthetic junction is 100 while the background is 0 , and the vessel radii are 4, 3 and 2 voxels. The image has been convolved with a Gaussian kernel of standard deviation 1 and a white Gaussian noise of standard deviation 30 has been added (top left of fig. 3). Top row of fig. 11 shows results of $\mathbf{e}_{g}$ in white and $\mathbf{e}_{m}$ in black on the left, computed with $\sigma=2$, and $\mathbf{e}_{\lambda_{1}}$ in white and $\mathbf{e}_{\lambda_{3}}$ in black on the right, computed with $\rho_{0}=\pi / 2$ and $B=2$. For both direction estimations, the results on the two widest vessels are very similar. However, the orientations are different near the junction and on the smallest vessel. On the one hand, the top white square on the two images shows points of the surface where $\mathbf{e}_{g}$ gives much better results than $\mathbf{e}_{\lambda_{1}}$. In this case, while the vectors $\mathbf{e}_{g}$ are well oriented, the vectors $\mathbf{e}_{\lambda_{1}}$ are parallel to the point of view of the projection and seem very small. This can be explained by a global deviation of $\mathbf{e}_{\lambda_{1}}$ in the direction orthogonal to the $3 \mathrm{D}$ plane that contains the junction, which is globally the direction of maximal intensity variation. On the other hand, the bottom small white square shows that the black vectors $\mathbf{e}_{\lambda_{3}}$ are better oriented than $\mathbf{e}_{m}$, due to the Gaussian smoothing that displaces the contour position. Globally, the basis of the gradient direction and the principal curvature directions give a better orientation estimation than the basis of the structure tensor eigenvectors.

In the second case, we present a synthetic hip joint (see bottom row of fig. 3) with a sphere and a semi-cylinder that simulate femoral head and hip. This $3 \mathrm{D}$ image has a size of $40 \times 40 \times 23$ and a $1 \times 1 \times 3.04$ voxel size. It has been generated with a binary image using the same parameter values as the synthetic junction and a noise standard deviation of 10 . We observe (fig. 1 bottom row) a better orientation of $\mathbf{e}_{\lambda_{1}}$, computed with $\rho_{0}=\pi / 4$ and $B=2$, (see XY slice and circle in the YZ slice) than $\mathbf{e}_{g}$, computed with $\sigma=1$. This better definition of the local structure is due to the fact that the gradient is very weak between the sphere and the semi-cylinder, so the normalized gradient orientation is not accurate. However, the tensor structure responds well in this case and points in the right direction, because it averages the orientations without taking into account opposite directions.

\section{Restoration}

An intuitive analogy can be made between the two filtering techniques. First, the low pass filter used by the first method is a heat diffusion equation with a data attachment, while the second method uses a Wiener filter, which also has lowpass characteristics. Second, anisotropic diffusion uses a diffusion function based on a threshold parameter $\delta$ on the first derivative of the intensity in the gradient direction to decide whether a region should be enhanced or smoothed. The proposed adaptive filtering uses a mapping function based on the first eigenvalue of the structure tensor to decide whether high frequencies should be increased or decreased. However, the PDE scheme progressively cools the solution while the 
adaptive filter obtains it in a single step. In this section, we compare restoration of noisy synthetic and real images using both methods.

\subsection{Synthetic Images}

Three synthetic images were created. The first synthetic image is a $3 \mathrm{D}$ image of size $15 \times 15 \times 60$ containing two bar-like structures of width 3 voxels separated by 2 voxels. This image varies only along $z$ axis and we represented an intensity profile at the center of $X Y$ plane and along $Z$ axis in figure 2] The image
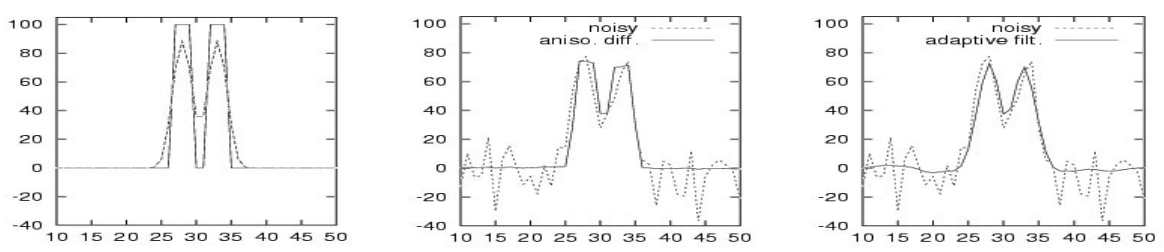

Fig. 2. Intensity profiles on a synthetic image.

was first convolved with a Gaussian of standard deviation 1 and then Gaussian white noise of standard deviation 15 was added. The solid line in the left graph in figure 2 shows the intensity profile of the binary image that we created, and the dashed line shows the convolved image. The middle graph in Figure 2 shows the restored image after anisotropic diffusion $(\sigma=1, \delta=7, \beta=0.05, \alpha=1)$ superimposed on the noisy image. Then, the right-hand graph in figure 2 shows the noisy image in slashed line and the restored image after adaptive filtering $\left(\rho_{0}=\pi / 3, B=2, \alpha=\gamma=0, \beta=10, \sigma=0.9\right)$ in plain line. We remark that the adaptive filtering tends to give a result that is closer to the convolved image, and the anisotropic diffusion attempts to converge to a segmented image with constant areas. The second synthetic image (top row of
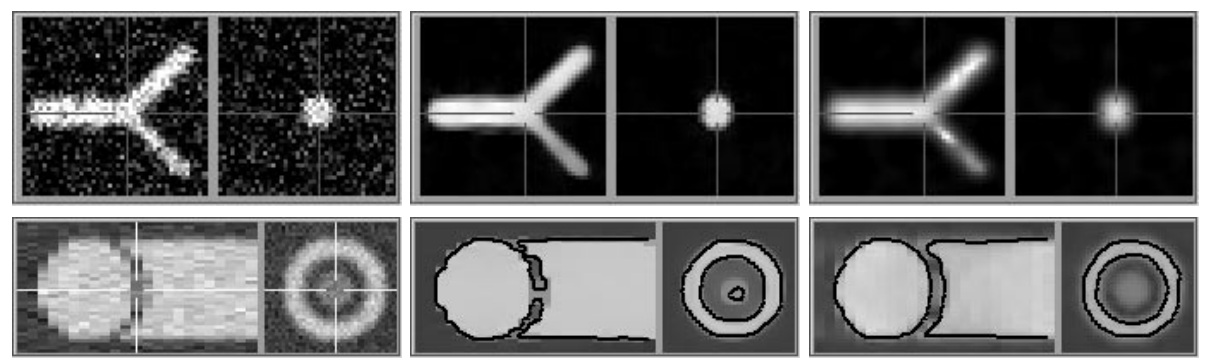

Fig. 3. Top row, comparison of the filtering on a synthetic $3 \mathrm{D}$ junction. Bottom row, comparison of the filtering on a synthetic 3D joint. Left, initial image with noise; middle, restoration using anisotropic diffusion; right, restoration using adaptive filtering. 
fig. 3) represents a 3D synthetic junction between vessels. This image was described in section 3 We use the following definition of the Signal to Noise Ratio (SNR): $S N R(I)=10 \log _{10} \frac{\sigma^{2}(I)}{\sigma^{2}\left(I_{b}-I\right)}$ where $I_{r}$ is the image to evaluate, $I_{b}$ is the initial binary image, and $\sigma$ denotes the standard deviation. Its value is 0.4 for the noisy image, 5.7 for the image restored by anisotropic diffusion $(\sigma=1, \delta=10, \beta=0.1, \alpha=1)$ and 2.0 for the image restored by adaptive filtering $\left(\rho_{0}=\pi / 2, B=2, \alpha=\gamma=0, \beta=10, \sigma=0.9\right)$. This means that we can obtain a better restoration using the anisotropic diffusion which corresponds to the visual impression in fig. 3, and which can be explained by the better estimation of the directions using the gradient and the principal curvature directions. The third synthetic image (bottom row of fig. 3), described in section 3 represents a $3 \mathrm{D}$ synthetic joint. The parameters used for the anisotropic diffusion are $\sigma=1, \delta=10, \beta=0.05, \alpha=1$ and for the adaptive filtering are $\rho_{0}=\pi / 4, B=2, \alpha=\gamma=0, \beta=2, \sigma=0.9$. An iso-intensity contour of threshold 60 was superimposed in black on the restored images to show that the adaptive filtering allows a better contrast enhancement. This is coherent with the better estimation of the orientations obtained by the structure tensor.

\subsection{Real Images}

Figure 4 presents results on two real images. The top row is a XY slice representation of a $94 \times 52 \times 26$ sub-volume from a 3D Magnetic Resonance Angiography. The voxel size is $0.93 \times 0.93 \times 1.5 \mathrm{~mm}$. We remark that the anisotropic diffusion $(\sigma=1, \delta=5, \beta=0.2, \alpha=0.3)$ provides a more homogeneous background than the adaptive filtering $\left(\rho_{0}=\pi / 2, B=2, \alpha=\gamma=0.5, \beta=1.5, \sigma=0.5\right)$, while the conservation of the small vessels is similar. This conservation depends on the parameters of each method. The bottom row presents a $85 \times 83 \times 20$ sub-volume of a hip joint CT scan of voxel size $0.82 \times 0.82 \times 3 \mathrm{~mm}$. We display on the top left of each image a zoom on the joint region that confirms that better enhancement is obtained by adaptive filtering $\left(\rho_{0}=\pi / 4, B=2, \alpha=\gamma=0.5, \beta=1.5, \sigma=0.1\right)$ compared to anisotropic diffusion $(\sigma=1, \delta=30, \beta=0.1, \alpha=1)$ as in the synthetic case.

\section{Discussion and Future Work}

We presented a comparison between two different types of anisotropic filtering. The first one uses the gradient and the principal curvature directions to steer the diffusion and the second one uses the eigenvectors of the structure tensor to weight the frequencies. We first compared the orientations used by each method and showed on synthetic 3D images that the gradient and principal curvature directions are better oriented in the case of vascular structures while the eigenvectors of the structure tensor respond better in regions of closed structures. We also made a qualitative comparison between the filtering results on both synthetic and real images applied to vessels and hip joint. As far as we know, 

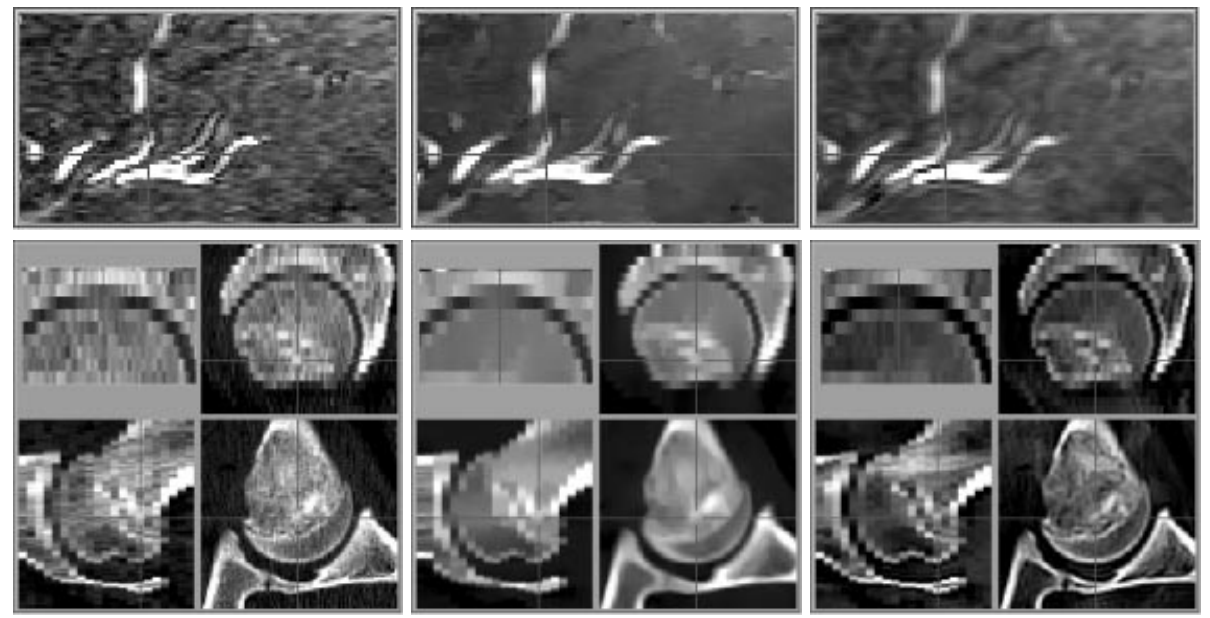

Fig. 4. Top row, 3D Magnetic Resonance Angiography. Bottom row, hip joint CT scan. Left, initial real image; middle, restoration using anisotropic diffusion; right, restoration using adaptive filtering.

this work is the first comparison that has been done between these two distinct filtering methods. An extended version of this work, including quantitative evaluation on synthetic images, is presented in [8]. In future work we also plan to use the same best estimation of orientation in boths methods, according to the application, and quantify the restoration of real images based on a specific segmentation algorithm and manually segmented images from physicians.

\section{Acknowledgements}

Thanks to L. Alvarez for supporting the second author as a post-doctoral grant, and to Miguel Aleman and Lauren O'Donnell for their help editing this paper.

\section{References}

[1] Abramatic J.F. and Silverman M. Nonlinear restoration of noisy images. IEEE Trans. of Pattern Analysis and Machine Intelligence, 4(2):141-149, 1982.

[2] Bigün J. and Granlund G. H. and Wiklund J. Multidimensional orientation: texture analysis and optical flow. IEEE Transactions on Pattern Analysis and Machine Intelligence, PAMI-13(8), August 1991.

[3] Knutsson H., Haglund L., Bårman H. and Granlund G. H. A framework for anisotropic adaptive filtering and analysis of image sequences and volumes. In Proceedings ICASSP-92, San Fransisco, CA, USA, March 1992. IEEE.

[4] Knutsson H., Wilson R. and Granlund G. H. Anisotropic non-stationary image estimation and its applications-part i: Restoration of noisy images. IEEE Trans. on Communications. COM-31, 3:388-397, 1983. 
[5] Krissian, K. Flux-based anisotropic diffusion: application to enhancement of 3d angiographies. Technical Report 0011, Instituto Universitario de Ciencias y Tecnologías Cibernéticas, Las Palmas, Spain, Dec. 2000.

[6] Krissian, K. Traitement multi-échelle: applications à l'imagerie médicale et à la détection tridimensionnelle de vaisseaux. $\mathrm{PhD}$ thesis, Univ. de Nice-Sophia Antipolis, Av. Joseph Vallot, 06108 Nice cedex 2, 2000.

[7] Krissian, K. and Malandain, G. and Ayache, N. Directional anisotropic diffusion applied to segmentation of vessels in $3 \mathrm{~d}$ images. In Scale-Space Theory in Computer Vision (Scale-Space), volume 1252 of Lecture Notes in Computer Science, pages 345-348, Utrecht, The Netherlands, July 1997. Springer Verlag.

[8] Krissian K., Rodriguez-Florido M.A., Ruiz-Alzola J., Westin C.-F. Comparison between two multidimensional anisotropic filtering techniques. Technical Report 18, Instituto Universitario de Ciencias y Tecnologías Cibernéticas, Las Palmas, Spain, 2001.

[9] Perona, P. and Malik, J. Scale-Space and edge detection using anisotropic diffusion. IEEE Trans. on Pattern Analysis and Machine Intel., 12(7):629-639, July 1990.

[10] Schriber W.F. Wirephoto quality improvement by unsharp masking. J.Pattern Recognition, 2:117-121, 1970.

[11] Weickert, J. Anisotropic Diffusion in image processing. Teubner-Verlag, Stuttgart, 1998.

[12] Westin C.-F. and Bhalerao A. and Knutsson H. and Kikinis R. Using Local 3D Structure for Segmentation of Bone from Computer Tomography Images. In CVPR, pages 794-800, Puerto Rico, June 1997.

[13] Westin C.F., Richolt J., Moharir V., Kikinis R. Affine adaptive filtering of CT data. Medical Image Analysis, 4:161-177, 2000. 